\title{
Interstitial Lung Disease and Pulmonary Arterial Hypertension in Overlap Syndrome: A Case Report
}

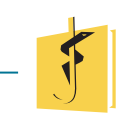

\author{
Mika Ana S. Frio, M.D.' ${ }^{1}$ \\ Sandra V. Navarra, M.D. ${ }^{2}$
}

\begin{abstract}
OBJECTIVE To present the onset of severe pulmonary arterial hypertension (PAH) in a patient with interstitial lung disease (ILD) associated with overlap syndrome.
\end{abstract}

CASE PRESENTATION A 42-year-old female was diagnosed with overlap syndrome consisting of systemic lupus erythematosus (SLE), systemic sclerosis (SSc) and rheumatoid arthritis (RA). The serologic profile included positive antinuclear antibody (ANA), anti-dsDNA, anti-RNP, anti-Ro, anti-Scl70, anti-Sm, rheumatoid factor and hypocomplementemia (C3, C4). She had chronic stable ILD for 17 years maintained on hydroxychloroquine (HCQ), prednisone $5 \mathrm{mg} /$ day and indacaterol. The current admission was due to progressive dyspnea and right-sided heart failure over the past month. Chest radiograph showed pulmonary congestion, and 2-dimensional echocardiography (2DE) disclosed severe $\mathrm{PAH}$ with systolic pulmonary arterial pressure (SPAP) of $76 \mathrm{mmHg}$ by tricuspid regurgitation (TR) jet, dilated right ventricle (RV) with poor systolic function, moderate pericardial effusion with no signs

Mika Ana S. Frio

mika_ana@yahoo.com

Section of Rheumatology

University of Santo Tomas Hospital

España, Manila, Philippines

2 University of Santo Tomas Hospital

España, Manila, Philippines of tamponade. She received furosemide, beraprost, sildenafil, and prednisone was increased to $20 \mathrm{mg} /$ day. Two weeks following discharge, there was complete resolution of symptoms and repeat 2DE showed non-dilated RV with good systolic function, normal SPAP of $21.4 \mathrm{mmHg}$ and minimal pericardial effusion. Prednisone was tapered to $5 \mathrm{mg} /$ day; beraprost, sildenafil and $H C Q$ were continued.

CONCLUSION Overlap syndrome was diagnosed by the combination of clinical features and serology distinctive of SLE, SSc and RA. Her illness, particularly ILD, was adequately controlled over several years, until the recent onset of $\mathrm{PAH}$ complicated by right-sided heart failure. The dramatic response to high-dose steroids is more consistent with inflammatory vasculitis of SLE activity rather than fibrosis typical of SSc.

Keywords overlap syndrome, pulmonary arterial hypertension, interstitial lung disease, systemic lupus erythematosus, scleroderma

\section{INTRODUCTION}

Current classifications have defined six autoimmune connective tissue diseases (CTDs), systemic lupus erythematosus (SLE), systemic sclerosis/scleroderma (SSc/Scl), polymyositis (PM), dermatomyositis (DM), rheumatoid arthritis (RA) and Siögren's syndrome. All six are syndromes without any definite "gold standard" for diagnosis. In early stages, the diagnosis is not always so obvious as these syndromes often 
have common features such as Raynaud's phenomenon, arthralgias, myalgias and positive tests for antinuclear antibodies (ANA)[1]. Overlap syndromes are defined by a combination of features of more than one CTD present in the same patient and often defined by a specific serological test. The diversity of clinical appearance precludes reliable estimates addressing the prevalence of overlap syndromes[2].

Pulmonary arterial hypertension (PAH) is a wellknown and severe complication associated with various CTDs, and occurs in approximately $10 \%$ of patients with SSc $[3,4,5]$. It may develop in isolation or in association with interstitial lung disease (ILD)[6].

A case of severe PAH in a patient with ILD associated with overlap syndrome is presented.

\section{CASE REPORT}

A 42-year-old Filipino female was diagnosed with overlap CTD (SLE, SSc, RA) after presenting with swollen hands, polyarthritis, Raynaud's phenomenon, skin tautness, alopecia, malar rash, anemia and hypocomplementemia (C3 and C4). Serology was positive for ANA > 1:2560 speckled, anti-DNA topoisomerase I (anti-Scl 70), anti-Smith (anti-Sm) 1:400, anti-ribonucleoprotein (anti-RNP) 1:3200, anti-Ro, anti-dsDNA $49.6 \mathrm{lU} / \mathrm{ml}$, anticardiolipin lgG $47 \mathrm{U} / \mathrm{ml}, \lg M 69 \mathrm{U} / \mathrm{ml}$ and rheumatoid factor 160 $\mathrm{IU} / \mathrm{ml}$. She developed a month-long history of productive cough, dyspnea, low-grade fever, bibasal crackles, wheezing and hemoptysis. Chest radiograph showed pulmonary infiltrates and bronchiectasis. Pulmonary function testing (PFT) revealed severe obstructive airway disease and probable peripheral airway restriction (forced vital capacity [FVC] 3 L, $59 \%$ predicted, forced expiratory volume in the first second of expiration [FEV1] 2 L, 53\% predicted, FEV 1/FVC 0.67). High-resolution computed tomography $(\mathrm{HRCT})$ revealed bilateral ground-glass opacities, bronchiectasis and subpleural blebs (Figure 1). She improved on indacaterol inhaler while being maintained on prednisone $5 \mathrm{mg} /$ day and $\mathrm{HCQ}$.

A few months ago, she was admitted for worsening exertional dyspnea, two-pillow orthopnea, cough, perioral cyanosis and bipedal edema. Chest radiograph showed pulmonary congestion. Echocardiography disclosed severe pulmonary arterial hypertension with systolic pulmonary arterial pressure (SPAP) $76 \mathrm{mmHg}$ by tricuspid regurgitation (TR) jet, dilated right ventricle (RV) with poor systolic func- tion and moderate pericardial effusion with no signs of tamponade. She received furosemide and was treated with piperacillin-tazobactam and amikacin. Prednisone was increased to $20 \mathrm{mg} /$ day and she was given beraprost and sildenafil. Two weeks later, there was complete resolution of symptoms. Repeat echocardiogram showed non-dilated RV with good systolic function, normal SPAP of $21.4 \mathrm{mmHg}$ and minimal pericardial effusion. Prednisone was tapered to $5 \mathrm{mg} /$ day. Beraprost, sildenafil and $\mathrm{HCQ}$ were continued.

\section{DISCUSSION}

Autoimmune rheumatic diseases are classified using internationally accepted criteria, frequently incorporating the detection of specific autoantibodies as unique diagnostic markers. Anti-dsDNA and anti-Sm have been used for establishing a diagnosis of SLE; rheumatoid factor for RA; anti-centromere (ACA), anti-Scl 70, and anti-RNA polymerase I and III antibodies for SSc/Scl [7].

Overlap syndromes or the presence of a second CTD can occur in $30 \%-52 \%$ of patients who have a diagnosis of SLE, RA and SSc/Scl [8].The proportion of patients described as having scleroderma overlap syndromes is greater $[9,10]$. Unlike Scl overlaps, well-defined overlap syndromes with SLE are unusual. The one exception is the overlap of RA and SLE, commonly referred to as "rhupus," which occurs in approximately $10 \%$ of SLE patients [1]. Having more than two CTDs existing in a single patient is more infrequent. Thorne and Piercy [1 1] described limited cutaneous SSc/Scl with overlap PM and inflammatory arthritis in a pregnant patient with mild ILD. Turkcapar et al. [12] were able to describe a patient with five coexisting autoimmune conditions with a fatal outcome.

The present patient's clinical features and serology are consistent with SLE, SSc and RA overlap. With corticosteroids and the addition of a disease-modifying antirheumatic drug (DMARD), a stable disease was achieved.

Often presenting with chronic symptoms of dyspnea and cough, ILD is the primary pulmonary manifestation of CTDs $[13,14]$. A Hungarian cohort of SSc-RA overlap described a frequency of $77 \%$ developing pulmonary fibrosis [15]. Physical examination may reveal inspiratory crackles, and PFT results demonstrate restrictive physiology often 
with reduced diffusing capacity. HRCT scanning is generally sufficient to confirm the diagnosis of ILD, although, in a minority of cases, surgical lung biopsy may be required [14]. Pulmonary fibrosis in the setting of SSc-associated ILD carries a poor prognosis. The only therapy known to influence survival is orthotopic lung transplantation [16].

Patients with SSc-PAH have a worse prognosis compared to patients with other forms of $\mathrm{PAH}$ with one-year survival rates ranging from 1\%-50\% [6]. Even when compared to patients with other forms of CTD-PAH, survival is markedly reduced in SSc-PAH. On the other hand, SLE-related PAH occurs less frequently compared to SSc. The mechanisms involved are not completely understood, however, PAH is thought to occur secondary to vasculitis, pulmonary embolism or ILD. PAH affects about $0.5 \%-14 \%$ of patients with SLE. Survival, which was previously quite poor even when compared to SSc-PAH, is now improved and better than in SSc-PAH with estimates at $75 \%$ if given $\mathrm{PAH}$-specific therapy [5]. In scleroderma-SLE overlap patients, the development of PAH is more frequent. However, if it can be ascribed to SLE, prompt immunosuppression with corticosteroids and cytotoxic drugs along with vasodilators can greatly improve prognosis [9].
A retrospective study described treatment with PAH-specific therapies, bosentan and sildenafil for 13 CTD-PAH-ILD patients, among which four had overlap syndromes. The survival estimate at a median duration of 34 months was $85 \%$ with mortality rates greater among patients with $\mathrm{SSc}$ versus other CTD subtypes [6].

The dramatic response to high-dose steroids is more consistent with inflammatory vasculitis of SLE activity rather than fibrosis typical of SSc. As discussed, this type of PAH has a better prognosis compared to SSc-PAH.

\section{CONCLUSION}

Overlap syndrome was diagnosed by a combination of clinical features and serology distinctive of SLE, SSc and RA. Her illness, particularly ILD, was adequately controlled over several years while being maintained on prednisone $5 \mathrm{mg} /$ day and $\mathrm{HCQ}$, until the recent onset of PAH complicated by right-sided heart failure. The dramatic response to high-dose steroids is more consistent with inflammatory vasculitis of SLE activity rather than fibrosis typical of SSc. 


\section{Declaration of Conflicting Interests}

An abstract of this article has been accepted for poster presentation in APLAR 2018, Kaohsiung, Taiwan. The authors report no other potential conflict of interest relevant to the article that may interfere with the presentation, review or publication of this case.

\section{REFERENCES}

1. Firestein GS, Gabriel SE, Mclnnes IB, O'Dell JR. Kelley and Firestein's Textbook of Rheumatology. 2017.

2. Gaubitz M. Epidemiology of connective tissue disorders. Rheumatology. 2006;45(3):iii3-iii4.

3. Kahler C. Pulmonary arterial hypertension (PAH) in connective tissue diseases. Rheumatology. 2006 Oct 1;45(3):iii $11-$ iii 13.

4. Condliffe R, Howard LS. Connective tissue disease-associated pulmonary arterial hypertension. F1000Prime Rep [Internet]. [cited 2015 Jan 5;7]. Available from: https:// www.ncbi.nlm.nih.gov/pmc/articles/PMC4311276/

5. Mathai SC, Hassoun PM. Pulmonary arterial hypertension in connective tissue diseases. Heart Fail Clin. 2012 Jul;8(3):413-25.

6. Mittoo S, Jacob T, Craig A, Bshouty Z. Treatment of pulmonary hypertension in patients with connective tissue disease and interstitial lung disease. Can Respir J, J Can Thorac Soc. 2010;17(6):282-6.

7. Jury E, D'Cruz D, Morrow W. Autoantibodies and overlap syndromes in autoimmune rheumatic disease. J Clin Pathol. 2001 May; 54(5):340-47.

8. Lockshin MD, Levine $A B$, Erkan D. Patients with overlap autoimmune disease differ from those with "pure" disease. Lupus Sci Med. 2015 May 1;2(1):e000084.

9. Sharma S, Kumar U. Scleroderma overlap syndromes. Int J Rheum Dis. 2016 Sep 1;19(9):831-3.

10. Horimoto AMC, da Costa IP. Overlap between systemic sclerosis and rheumatoid arthritis: a distinct clinical entity? Rev Bras Reumatol Engl Ed. 2016 Jul 1;56(4):287-98.
11. Thorne I, Piercy C. Early scleroderma overlap syndrome (pulmonary hypertension, ILD, polymyositis and inflammatory arthritis) and an unplanned pregnancy. Rheumatology Advances in Practice. 2017 Oct; 1(1).

12. Turkcapar N, Olmez U, Ozer D, Duzgun N, Duman M. A case of overlap syndrome with rheumatoid arthritis, systemic lupus erythematosus, systemic sclerosis and secondary Siögren's syndrome. Rheumatol Int. 2006 Jul;26(9):841-5.

13. Shaw M, Collins BF, Ho LA, Raghu G. Rheumatoid arthritis-associated lung disease. Eur Respir Rev. 2015 Mar $1 ; 24(135): 1-16$.

14. Kim EJ, Collard HR, King TE. Rheumatoid arthritisassociated interstitial lung disease. Chest. 2009 Nov; 136(5): 1397-405.

15. Szücs G, Szekanecz Z, Zilahi E, Kapitány A, Baráth S, Szamosi $S$, et al. Systemic sclerosis-rheumatoid arthritis overlap syndrome: a unique combination of features suggests a distinct genetic, serological and clinical entity. Rheumatology. 2007 Jun 1;46(6):989-93.

16. Herzog EL, Mathur A, Tager AM, Feghali-Bostwick C, Schneider F, Varga J. Interstitial lung disease associated with systemic sclerosis and idiopathic pulmonary fibrosis. Arthritis Rheumatol. Hoboken, NJ. 2014 Aug;66(8):1967-78.

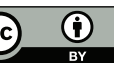

Open Access This article is licensed under a Creative Commons Attribution 4.0 International License, which permits use, sharing, adaptation, distribution and reproduction in any medium or format, as long as you give appropriate credit to the original author(s) and the source, provide a link to the Creative Commons license, and indicate if changes were made. The images or other third party material in this article are included in the article's Creative Commons license, unless indicated otherwise in a credit line to the material. If material is not included in the article's Creative Commons license and your intended use is not permitted by statutory regulation or exceeds the permitted use, you will need to obtain permission directly from the copyright holder. To view a copy of this license, visit http://creativecommons.org/licenses/ by/4.0/. 